\title{
EVALUACIÓN DE LA ACEPTACIÓN DE DISTINTAS PRESENTACIONES DE UN CONTRACEPTIVO ORAL EN PALOMAS TORCAZAS
}

\author{
Olivera, L. ${ }^{1} ;$ Rodríguez, E²; Pereyra, S. ${ }^{3}$; \\ SaWchiK, J. ${ }^{3}$; Ceretta, S. ${ }^{3} \&$ Banchero, G. ${ }^{3}$
}

\section{RESUMEN}

Las palomas torcazas (Zenaida auriculata) causan pérdidas que pueden representar hasta $50 \%$ de daño en algunos cultivos. El objetivo fue medir la aceptación de cebos contraceptivos para palomas torcazas en jaulas individuales (10 aves/tratamiento). Luego de la cuarentena y la aclimatación de las aves a la jaula, se les ofreció durante cuatro horas diarias por 21 días consecutivos tres presentaciones (tratamientos) del pellet con nicarbazina al 0,5\%: (1) OvoControlS ${ }^{\circledR}$ diseñado para gorriones; (2) mezcla de OvoControl $\AA \AA$ con maíz picado en proporción 78:22 y (3) OvoControlP $\AA$ partido, diseñado para paloma doméstica (Columba livia). En la mañana se ofreció a cada ave el cebo del tratamiento, y por la tarde se ofreció dieta de mantenimiento en igual cantidad, y se registró lo ingerido. El consumo promedio/semana de OvoControlS ${ }^{\circledR}$ y de la mezcla OvoControlS ${ }^{\circledR}$ con maíz fue menor a 1,0g. El OvoControlP ${ }^{\circledR}$ partido fue aceptado por las aves. El consumo aumentó en las semanas 2 y $3(\mathrm{p}<0,05)$, superando $1,0 \mathrm{~g}$ promedio/semana y fue mayor que las otras presentaciones $(\mathrm{p}<0,05)$. Se puede concluir que esta presentación de nicarbazina al $0,5 \%$ fue aceptada y consumida por palomas torcazas en condiciones de cautiverio, en cantidades que podrían causar un efecto en la reproducción de la especie.

Key words: Zenaida auriculata, control reproductivo, Ovocontrol, nicarbazina.

1.- Contratado por proyecto marco del Acuerdo Instituto Nacional de Investigación Agropecuaria - Dirección General de Servicios Agrícolas. Av. Millán 4703. Montevideo. Uruguay. Tel: (+598) 23098410.

Email: lourdes.m.olivera@gmail.com

2.- Dirección General de Servicios Agrícolas. Av. Millán 4703. Montevideo. Uruguay. Tel: (+598) 23098410.

3.- Instituto Nacional de Investigación Agropecuaria. Ruta 50, Km 11. Colonia, Uruguay. Tel: (+598) 45748000. Manuscrito recibido el 30 de diciembre de 2019 y aceptado para su publicación el 14 de mayo de 2020.

Olivera, L.; Rodríguez, E.; Pereyra, S.; Sawchik, J.; Ceretta, S.; Banchero, G. Evaluación de la aceptación de distintas presentaciones de un contraceptivo oral en palomas torcazas.

FAVE - Ciencias Agrarias 19 (1): 55-66. CC BY-NC-SA 4.0 


\section{ABSTRACT \\ Evaluation of different presentation's acceptance of an oral contraceptive in eared doves.}

Eared doves (Zenaida auriculata) may cause up to $50 \%$ of losses in some damaged crops. The objective of this study was to measure a contraceptive bait (nicarbazin pellets $0,5 \%$ ) acceptance by Eared Doves in individual cages (10 birds/treatments). After quarantine and an adaptation period to the cages, the bait (nicarbazin pellets $0,5 \%$ ) was offered to the doves for four hours per day for 21 consecutive days in three formulations (treatments) according to the bait: (1) OvoControlS ${ }^{\circ}$ developed for sparrows; (2) OvoControlS ${ }^{\circledR}$ mixed with cracked corn in a 78:22 ratio; (3) was offered the corresponding bait treatment, cracked OvoControlP ${ }^{\circledR}$ elaborated for rock pigeon (Columba livia). Every morning each bird was offered the bait of the treatment, and in the afternoon a maintenance diet was offered in the same amount and daily consumption was registered. The average consumption/week

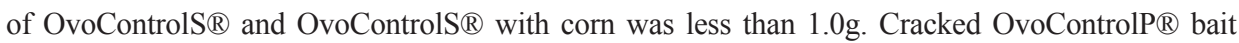
was accepted by birds. The consume increased in weeks 2 and $3(\mathrm{p}<0.05)$, exceeding $1.0 \mathrm{~g}$ average/ week and was higher than the other formulations $(\mathrm{p}<0.05)$. It can be concluded that this treatment was accepted and consumed by eared doves in captivity in amounts that could cause an effect on this species reproduction.

Key words: Zenaida auriculata, reproductive control, Ovocontrol, Nicarbazin.

\section{INTRODUCCIÓN}

En las últimas décadas la expansión agrícola y la incursión en la cría de ganado en confinamiento, ha aumentado la disponibilidad de alimento para las aves granívoras. En Uruguay, estos sistemas productivos se encuentran formando un paisaje en mosaico, alternados con parches de arbustos y montes naturales o implantados, así como fuentes de agua. La paloma torcaza (Zenaida auriculata) se adaptó a estos ambientes donde posee disponibilidad de alimento, agua y sitios para descansar y nidificar $(9$, 20), favoreciendo una explosión poblacional de la especie mencionada. Las palomas torcazas dañan cultivos de cereales y oleaginosos, además de producir pérdidas en establecimientos con ganado en confinamiento. Por ejemplo, se reportaron pérdidas en plántulas de soja de hasta un $49 \%$ y
$9 \%$ en bordes y centro de chacras y el daño consistía en la pérdida total o parcial de los cotiledones (5).

Desde la década de los $80^{\prime}$, las estrategias de manejo se enfocaron en las medidas de prevención de los daños y protección del cultivo, desarrollando e implementando métodos de repelencia física y química (15, 18, 20). Sin embargo, debido al aumento de las áreas productivas de las últimas décadas, se han incrementado los problemas de aves (especialmente de palomas torcazas). Además, estas medidas tienen sus limitaciones y a veces son difíciles de implementar, debido a problemas de aplicación y a la relación costo/ beneficio, entre otros. Por lo tanto, es necesario ampliar las estrategias que se utilizan para mitigar los daños.

Una herramienta de manejo implementada en Nueva Zelanda, Australia, Estados Unidos y algunos países de Europa, es la 
regulación de poblaciones utilizando métodos contraceptivos $(10,12,14)$. Desde la década de los $60^{\prime}$ estos químicos están siendo estudiados y se basan en la disminución de las tasas de nacimiento o reclutamiento (4). Desde sus comienzos, la investigación en contraceptivos estuvo orientada hacia mamíferos y es donde se han puesto más esfuerzos. En las aves, la investigación ha sido más restringida; y si bien se han propuesto diversos químicos, los estudios han estado centrados en el desarrollo de dos compuestos: 20,25-diazacolesteroldihidrocloruro (DiazaCon () y nicarbazina (OvoControl $®)(14,24)$. El 20,25-diazacolesteroldihidrocloruro fue probado en codorniz (Coturnix coturnix), disminuyendo en $85 \%$ de la producción de huevos (22); y en cotorra (Myiopsitta monachus), reduciendo la cantidad de huevos por nido de $3,6 \pm 1,1$ huevos/nido en parejas control a 1,6 $\pm 0,7$ huevos/nido en parejas tratadas (3). Por su parte, la nicarbazina administrada a palomas domésticas (Columba livia) redujo en $59 \%$ el número de pichones y disminuyó hasta el $56 \%$ en la eclosión de los huevos de gansos de Canadá (Branta canadensis) (2, 7, 23).

La nicarbazina es un esteroide utilizado en aves de corral desde los años 50' como droga para prevenir coccidios (11). Se trata de una sal bimodal que consiste en dos componentes: el 4, 4'dinitrocarbanilide (DNC) que es el componente activo y el hydroxy-4, 6-dimethylpyrimidine (HDP), que actúa como "adyuvante", incrementando su absorción intestinal (17). Su efecto contraceptivo fue descubierto en gallinas ponedoras donde se observó una reducción en la eclosión de los huevos entre el sexto y décimo día de tratamiento. Posteriormente, en 2005, se registró este ingrediente activo bajo el nombre de OvoControlG ${ }^{\circledR}$ para gansos de Canadá (Branta canadienses), y en 2007 para palomas domésticas con el nombre de OvoControlP ${ }^{\circledR}$ (11).
Un aspecto fundamental en el uso de esta herramienta de manejo es la administración correcta del producto a las aves, de forma que alcancen la dosis mínima necesaria para causar efecto en su ciclo reproductivo. Según estudios, la nicarbazina no es palatable para gansos de Canadá (6), lo que llevó a proponer modificaciones en las características del cebo (forma, composición y tamaño), para aumentar su atractividad $(1,6,24)$. Dentro de los cebos evaluados, se aplicó la nicarbazina a maíz picado, mezclada con aceite y leche en polvo (6). Si bien esta técnica aumentó la palatabilidad para los gansos, la formulación presentaba problemas de estabilidad de la molécula, al ser aplicada de forma tópica sobre el maíz, además de registrarse consumo por especies no-blanco. Otro tipo de cebo evaluado fue un pellet elaborado a base de harina de maíz e imitando el aspecto de éste grano, el cual tuvo una aceptación limitada en experimentos de campo y nula en semi-cautiverio. Finalmente, al utilizar un cebo formulado a base de harina de trigo, imitando pan, se obtuvo aceptación por parte de los gansos de Canadá en condiciones de laboratorio (6). En paloma doméstica, se estudiaron cuatro diferentes tipos de cebos con nicarbazina al $0,25 \%$, no encontrando diferencias significativas en su consumo ni alcanzando valores satisfactorios de DNC en sangre (1). Posteriormente se probaron tres nuevos cebos: dos formulados con $0,25 \%$ y uno con $0,5 \%$ de nicarbazina. Todos fueron evaluados con y sin recubrimiento de aceite. El consumo de los cebos recubiertos fue significativamente mayor que cuando eran presentados solos (1). Con la formulación de $0,5 \%$ se alcanzaron valores de DNC satisfactorios en sangre, suficientes como para producir un efecto en el ciclo reproductivo (1), cuando el consumo diario alcanzado fue $116,9 \pm 12,7 \mathrm{mg}$ de nicarbazina/Kg de masa corporal (2). 
En Uruguay, los primeros experimentos realizados con contraceptivos utilizaron aceite mineral para evitar la eclosión de huevos de cotorra (Myiopsitta monachus), realizándose pruebas exitosas en laboratorio y campo (19). En el año 1991, se evaluó el posible rol que los quimioesterilizantes podrían tener en un programa de manejo integrado de aves y se concluyó que, con los compuestos contraceptivos disponibles en ese momento, el método no sería efectivo para disminuir el daño inmediato (13). Al presente, no se encontraron antecedentes del uso de contraceptivos en paloma torcaza, ni en otras palomas del género Zenaida. Debido a los avances logrados en Estados Unidos sobre nuevos contraceptivos aplicados a otras especies de aves $(1,2,3,12)$, se propone evaluar en Uruguay el uso de nicarbazina, como una medida complementaria a las existentes, para la reducción del daño a mediano y largo plazo.

Debido a que la nicarbazina es excretada rápidamente del cuerpo del ave, es necesario que ingieran diariamente cantidades suficientes, para que el tratamiento sea efectivo. Esto es una ventaja para las especies no-blanco, ya que si consumen una cantidad de nicarbazina que produzca una disminución de sus parámetros reproductivos, el efecto desaparecerá cuando no ingiera el cebo (2). Esto genera el desafio de garantizar un consumo en días consecutivos de la especie problema, que debe ser tenido en cuenta para diseñar una estrategia de administración del producto (1).

El objetivo de este estudio fue medir la aceptación de tres diferentes presentaciones de cebo con nicarbazina (Ovocontrol ${ }^{\circledR}$ ) para palomas torcazas. La hipótesis de trabajo es que la nicarbazina a la dosis formulada $(0,5 \%)$, es palatable para las palomas torcazas en al menos una de las presentaciones evaluadas. Se asume en este trabajo como palatable aquel alimento con un con- sumo promedio semanal superior a $1,0 \mathrm{~g}$ (adaptado de Avery et al., 2006'1).

\section{MATERIALES Y MÉTODOS}

Los experimentos dieron inicio en diciembre de 2018 y finalizaron en abril de 2019, en los aviarios ubicados en la Dirección General de Servicios Agrícolas, Montevideo, Uruguay. Ambos aviarios poseen luz natural y temperatura controlada. Las evaluaciones constaron de tres etapas: 1- captura de las palomas, 2cuarentena, 3- experimentación.

La captura de las palomas se realizó en un tambo del departamento de Durazno $\left(33^{\circ} 20^{\prime} 27.58^{\prime \prime} \mathrm{S}, 56^{\circ} 33^{\prime} 50.40^{\circ} \mathrm{O}\right)$, Uruguay. Se utilizaron dos trampas de suelo de un metro cuadrado colocadas junto con el almacenaje de alimento para el ganado.

En la cuarentena, las palomas se ubicaron en jaulas individuales de $40,5 \mathrm{~cm}$ de largo, $23,5 \mathrm{~cm}$ de alto y $24,0 \mathrm{~cm}$ de profundidad, de malla de alambre de 1,5 por $13,0 \mathrm{~cm}$ de sección, un posadero, dos comederos y un bebedero. Esta etapa tuvo una duración de 22 días, durante la cual se les ofreció agua y alimento ad libitum, que consistió en partes iguales de trigo, sorgo y maíz picado. Se les suministró además antibióticos, antiparasitario y vitaminas. Esto se realizó para lograr un óptimo estado físico, similar en todas las aves y prevenir enfermedades en las etapas posteriores del experimento.

La etapa de experimentación se llevó a cabo en un aviario con jaulas de 1,20 x 1,20 $\mathrm{mts}$, con $0,85 \mathrm{mts}$ de profundidad, con un individuo por jaula. Esta incluye una primera fase de aclimatación, cuya duración fue de cinco días. Allí se midió el consumo diario de la dieta de mantenimiento por paloma, mientras se permitió a las aves que se habituaran al nuevo aviario. 
Para registrar cambios en el peso de la ración ocasionados por ganancia o pérdida de humedad, se colocó un comedero con la misma cantidad de alimento ofrecida a las aves como testigo negativo fuera del alcance de éstas. Se calculó el consumo de alimento, por paloma, por día como: $\mathrm{C}=$ Piaju-Pf, siendo Piaju el peso inicial en función de la variación medida en el testigo negativo y $\mathrm{Pf}$ el peso final del contenido remanente en el comedero agregando lo que el ave vuelca al alimentarse. A su vez, Piaju se calculó como (Pi * Pft)/Pit, siendo Pi el peso inicial del alimento, Pit el peso inicial testigo negativo, y Pft: peso final testigo negativo.

En la aclimatación, se calculó el consumo diario promedio (g/día) de las aves. A este valor se lo denominó consumo diario medido (CDM) de alimento por paloma. Luego, se promediaron los valores obtenidos en los cinco días de esta etapa.

Culminada la aclimatación, se ofreció a las aves el alimento a evaluar. Los productos utilizados fueron dos formulaciones de OvoControl ${ }^{\circledR}$ con una concentración de nicarbazina de $0,5 \%$. Ambos fueron formulados como pellets por Innolytics LLC (Rancho Mirage, Estados Unidos). El OvoControlP ${ }^{\circledR}$ está registrado para paloma doméstica (Columba livia) en todo Estados Unidos, con excepción del estado de New Hampshire. Su formulación es en forma de pellet cilíndrico de aproximadamente 7,0 $\mathrm{mm}$ de largo y 5,0 $\mathrm{mm}$ de diámetro. El OvocontrolS $\AA$ es una nueva formulación de contraceptivo para gorriones (Passer domesticus), también de forma cilíndrica, con tamaño de pellet de 1,5 $\mathrm{mm}$ de largo y de diámetro, aún en fase de experimentación en Innolytics.

Cada día, se ofreció $10 \mathrm{~g}$ de contraceptivo a cada ave en un comedero plástico de 8,5 $\mathrm{cm}$ de diámetro y 4,5 de alto a las 9:30 h. Esta cantidad corresponde al CDM promedio, medido en la aclimatación. Debajo de cada comedero, se colocó una bandeja para recoger lo que el ave puede volcar al alimentarse. A las 13:30 h se retiró el comedero con el remanente de contraceptivo de cada jaula y se registró su peso $(\mathrm{g})$. Durante este período las aves sólo tenían disponible para su consumo una de las presentaciones de contraceptivo a ser evaluadas. Desde las 13:30 h a las 8:00 h de la mañana se colocaba un comedero de iguales dimensiones conteniendo $10 \mathrm{~g}$ con dieta de mantenimiento.

A las 8:00 h del día siguiente, se retiraba el comedero con el remanente de la ración y se pesaba. A las 9:30 h se volvía a colocar el contraceptivo, al igual que el día anterior. Este proceso se repitió durante 21 días consecutivos. Al igual que en la etapa de aclimatación, se agregó un testigo negativo para registrar las fluctuaciones en el peso debidas a pérdida o ganancia de humedad. Este consistió en un comedero sin acceso a las aves, con las mismas dimensiones y contenido de cada uno de los alimentos evaluados.

En todos los tratamientos, se trabajó con un $n=10$ palomas. En el tratamiento 1, el alimento de prueba fue contraceptivo OvoControlS ${ }^{\circledR}$. En el tratamiento 2 se empleó una técnica sugerida por Avery y Wolf Innolytics-LLC (com. pers.) para hacer más atractivo el cebo. Ésta consistió en mezclar el contraceptivo OvoControlS ${ }^{\circledR}$ con granos de maíz picados, los cuales se incluyeron en una proporción 78/22 de contraceptivo y maíz respectivamente. Al retirar este alimento de las jaulas, se registró el remanente del comedero y posteriormente se apartó el maíz del contraceptivo para poder discriminar su consumo. En el tratamiento 3 el alimento evaluado fue OvoControlP $\AA$ partido. Para facilitar la ingestión por parte de las aves, en este último tratamiento los pellets fueron partidos mediante compresión hasta lograr partículas con un diámetro de entre 0,5 a 3,0 mm. 


\section{Olivera et al.}

Además, en la etapa de cuarentena previa, el contraceptivo también fue ofrecido a las aves junto con la ración de mantenimiento.

Posteriormente, se calculó el consumo diario (C) como se detalló previamente, para el contraceptivo y la ración de mantenimiento de cada paloma. Se empleó estadística descriptiva, promediando el CDM por semana para conocer si se alcanza el valor promedio semanal de 1,0 g definido como umbral para ser considerado un alimento palatable. Se presentan los resultados como medias diarias y semanales \pm desvíos estándar. Se comprobó el grado de ajuste de los datos a una distribución normal con el test de Shapiro-Wilk. Se realizó un Test de Friedman por no ajustarse los datos a una distribución normal, con la presentación del contraceptivo como principal efecto. Para discriminar las diferencias entre tratamientos y entre semanas, se aplicó la prueba de rangos con signo de Wilcoxon. Se trabajó con el paquete estadístico R (versión 3.4.3). En todos los casos se utilizó de referencia un $\alpha \leq 0,05$.

\section{RESULTADOS}

En el tratamiento 1, (OvoControlS $\AA)$ se encontró que solo dos palomas consumieron el cebo. Una de ellas ingirió en prome-

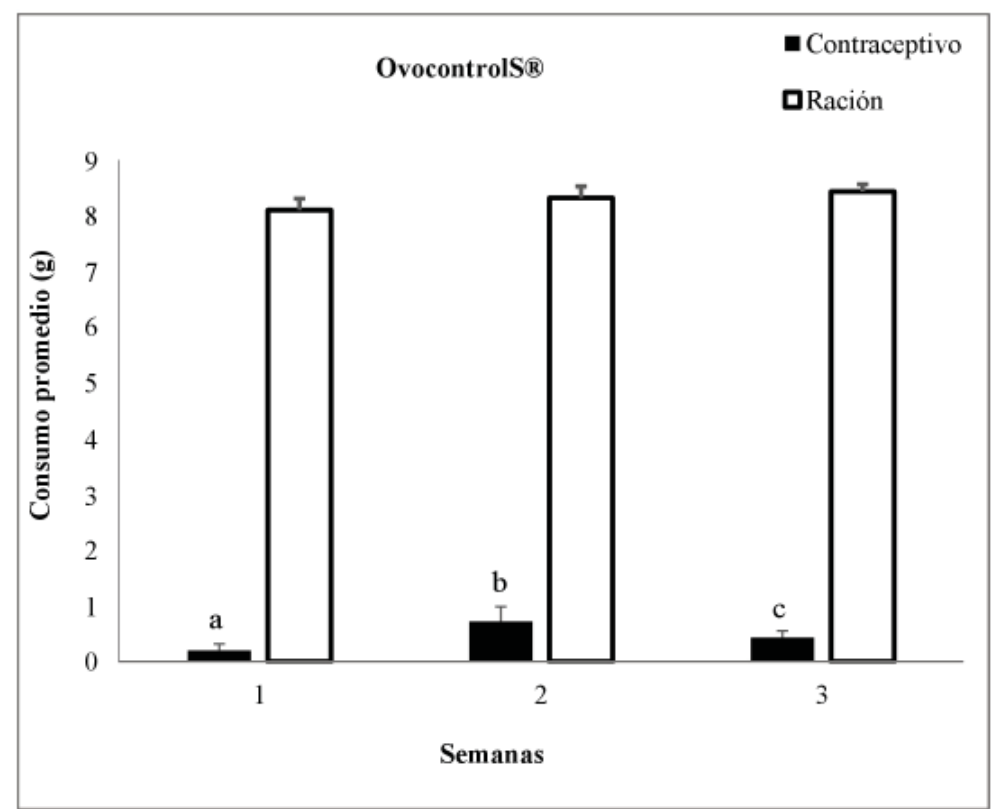

Figura 1. Consumo diario promedio ( $g$ ) y su desvio estándar del contraceptivo OvocontrolS® y ración de mantenimiento en las tres semanas del experimento para un $n=10$ palomas. El contraceptivo fue ofrecido diariamente durante cuatro horas, teniendo disponible ración de mantenimiento durante el resto del día. Letras distintas indican diferencias significativas entre semanas basadas en prueba de rangos con signo de Wilcoxon $(\alpha \leq 0,05)$. Figure 1. Daily mean consumption $(\mathrm{g})$ and standard deviation of Ovocontrol $\mathrm{B}$ and maintenance diet. The experiment lasted for three weeks and involves 10 eared doves. Contraceptive bait was offer daily during four hours. Maintenance diet was available the rest of the day. Different letters indicate significant differences among weeks based on signed rank test Wilcoxon $(\alpha \leq 0.05)$. 
dio $0,9 \pm 0,7 \mathrm{~g} /$ día a partir del día 6 y otra consumió $5,3 \pm 2,1 \mathrm{~g}$ /día a partir del día 9 .

Se registraron diferencias significativas entre semanas, siendo el mayor valor en la semana $2(\mathrm{p}<0,05)$. No obstante, ninguno de los valores promedios semanales fueron superiores a $1,0 \mathrm{~g}$ (Figura 1). Al comparar la ingesta de ración de mantenimiento, no se observaron diferencias significativas.

El consumo de la mezcla OvocontrolS ${ }^{\circledR}$ + maíz (tratamiento 2) incrementó en el tiempo (Figura 2), alcanzando su máximo en la tercera semana $(\mathrm{p}<0,05)$. Al discriminar el contraceptivo de la mezcla, se puede apreciar que su consumo acompañó esta tendencia, siendo significativamente mayor en la semana $3(p<0,05)$. Sin embargo, en esta última semana, la ingesta de las aves fue igual o menor a $0,09 \mathrm{~g} / \mathrm{ave} /$ día, resultando en un promedio semanal menor a $1,0 \mathrm{~g}$. El consumo de ración presentó el mismo patrón siendo significativamente mayor en la última semana $(\mathrm{p}<0,05)$.

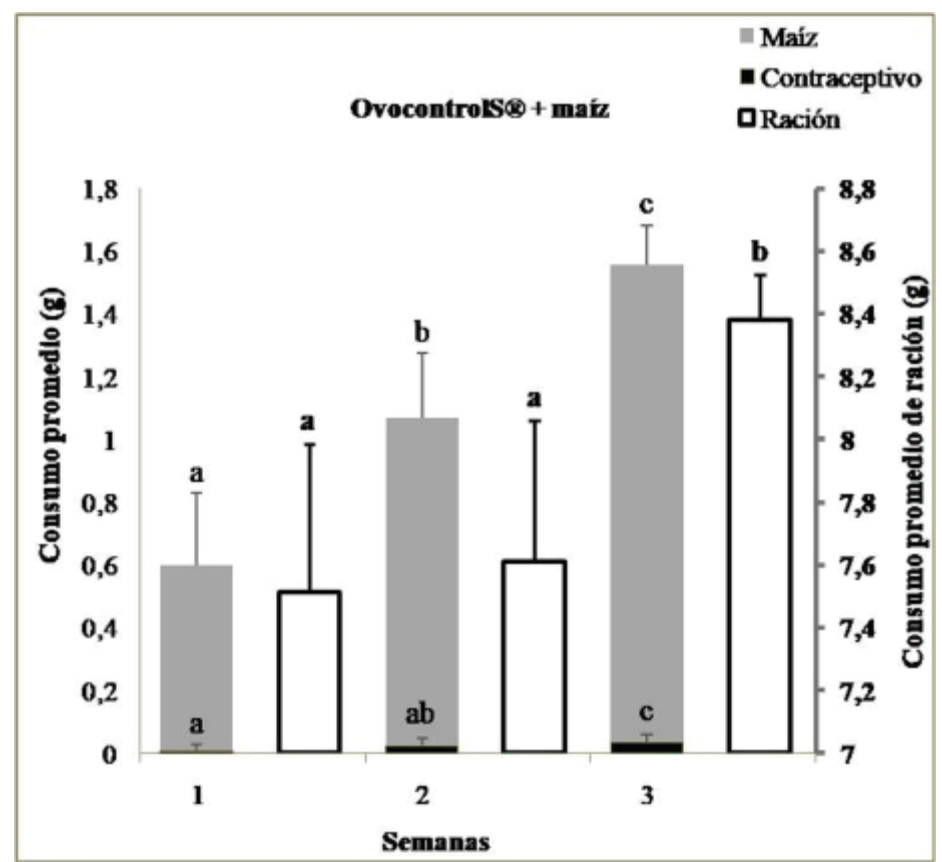

Figura 2. Consumo diario promedio (g) y su desvío estándar para la mezcla OvocontrolS ${ }^{\circledR}+$ maíz (discriminada la ingesta de contraceptivo del maiz) y de la ración de mantenimiento, durante tres semanas de experimento para un $n=10$ palomas. La mezcla Ovocontrol $(\circledR+$ maiz en el mismo comedero fue ofrecida diariamente durante cuatro horas, teniendo disponible ración de mantenimiento durante el resto del día. Letras distintas indican diferencias significativas entre semanas basadas en prueba de rangos con signo de Wilcoxon $(\alpha \leq 0,05)$. Figure 2. Daily mean consumption $(g)$ and standard deviation of OvocontrolS ${ }^{\circledR}$ + corn (contraceptive consumption differentiated from corn) and maintenance diet. The experiment lasted for three weeks and involve 10 eared doves. The mix Ovocontrol ${ }^{\circledR}+$ corn in the same feeder was offer daily during four hours. Maintenance diet was available the rest of the day. Different letters indicate significant differences among weeks based on signed rank test Wilcoxon $(\alpha \leq 0.05)$. 
El consumo promedio semanal de OvoControlP ${ }^{\circledR}$ partido (tratamiento 3, Figura 3), fue superior a $1,0 \mathrm{~g}$ y éste aumentó en el tiempo hasta ser significativamente mayor en las semanas 2 y $3(p<0,05)$. De manera consecuente, el consumo de ración disminuyó significativamente en las $2^{\mathrm{a}}$ y $3^{\mathrm{a}}$ semanas $(p<0,05)$.
Al comparar los resultados de las tres presentaciones del contraceptivo (Figura 4), se puede observar que el consumo del OvocontrolP ${ }^{\circledR}$ partido es superior al de las otras presentaciones ofrecidas $(p<0,05)$. Además, el consumo del OvoControlS ${ }^{\circledR}$ ofrecido en la mezcla con maíz (tratamiento $2)$, resultó en un menor consumo $(p<0,05)$ en relación al del contraceptivo ofrecido solo (tratamiento 1).

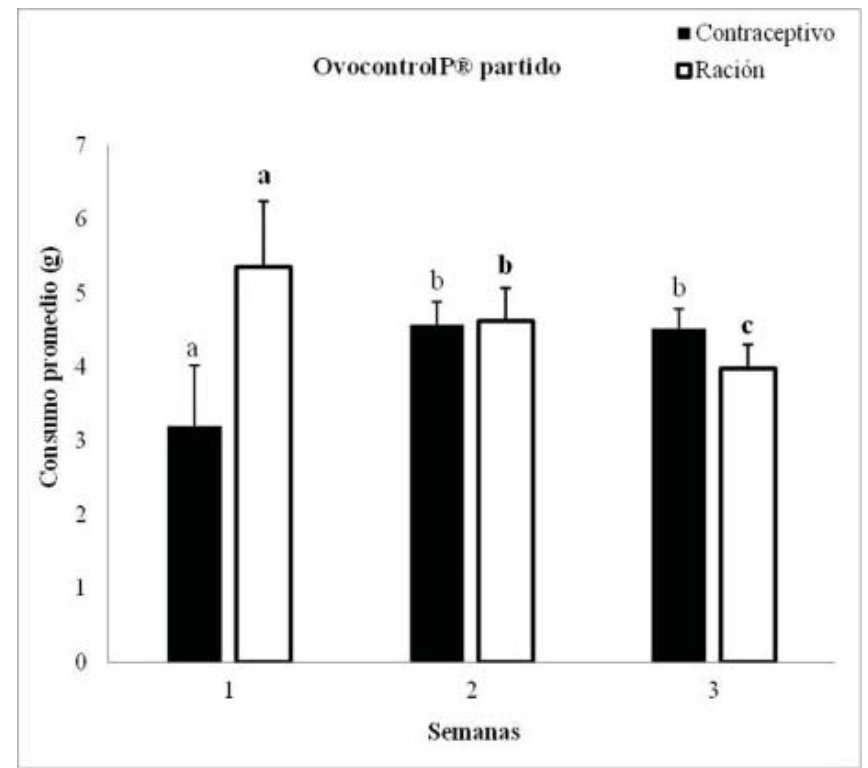

Figura 3. Consumo diario promedio ( $g$ ) y su desvio estándar para el contraceptivo OvocontrolP $\mathbb{}$ partido y la ración de mantenimiento en las tres semanas del experimento para un $n=10$ palomas. El contraceptivo fue ofrecido diariamente durante cuatro horas, teniendo disponible ración de mantenimiento durante el resto del día. Letras distintas indican diferencias significativas entre semanas basadas en prueba de rangos con signo de Wilcoxon $(\alpha \leq 0,05)$. Figure 3. Daily mean consumption ( $g$ ) and involved 10 eared doves. The experiment lasted three weeks and involves 10 eared doves. Contraceptive bait was offered daily during four hours. Maintenance diet was available for the rest of the day. Different letters indicate significant differences among weeks based on signed rank test Wilcoxon $(\alpha \leq 0.05)$. 
Evaluación de cebos con nicarbazina como contraceptivo en paloma torcaza

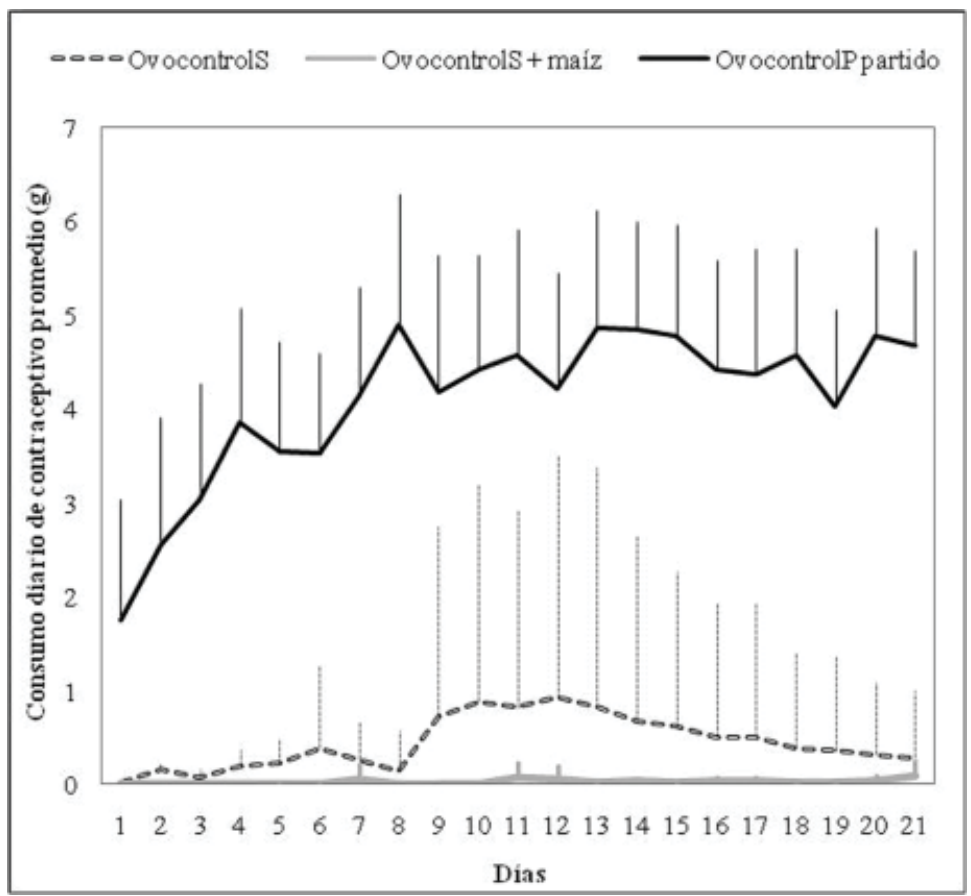

Figura 4. Consumos diarios promedio ( $g$ ) con sus respectivos desvios estándar, de las tres presentaciones de contraceptivo evaluadas. Cada alimento en estudio se ofreció diariamente a 10 palomas durante cuatro horas, teniendo disponible ración de mantenimiento durante el resto del día. Figure 4. Daily mean consumption ( $\mathrm{g}$ ) and standard deviation of three contraceptive formulations. Each treatment was daily offered to 10 eared doves during four hours. Maintenance diet was available the rest of the day.

\section{DISCUSION}

La hipótesis de trabajo fue aceptada, ya que la nicarbazina a la dosis formulada $(0,5 \%)$, es palatable para las palomas torcazas como cebo OvoControlP $®$ partido. Con la mencionada presentación, se logró un consumo por masa corporal del animal y por día, que podría llegar a disminuir la reproducción de éstas.

Asumiendo que en nuestro experimento una paloma torcaza pesa en promedio 123 g (8), podríamos estimar el consumo medio de nicarbazina en $166,9 \pm 33,4 \mathrm{mg}$ por kilo de masa corporal del ave por día. Este valor es superior al registrado para paloma doméstica, con la cual era necesario un consumo de $116,9 \pm 12,7 \mathrm{mg}$ de nicarbazina diario por kilo de masa corporal del ave (2).

Por otro lado, el consumo de OvocontrolS $\AA$ en sus dos presentaciones, fue menor al reportado por Avery et al. $\left(2006^{1}\right)$ para paloma doméstica. En estudios previos realizados con ración para ganado se determinó que las palomas torcazas eran capaces de buscar y consumir selectivamente algunos de los ítems que componían la dieta del ganado $(16,21)$. En ambos estudios se encontró que los granos partidos entre 2 y $3 \mathrm{~mm}$ presentaban un consumo preferencial. Este tamaño 
de partícula es superior al tamaño de los pellets de OvocontrolS ${ }^{\circledR}$. En ambos experimentos con este último contraceptivo, la ingesta de dieta de mantenimiento (un alimento conocido y aceptado por las aves), no varía o incluso aumenta en el transcurso de las semanas. Esto último indicaría que, en ambos casos, rechazan el contraceptivo en las horas de la mañana, esperando para consumir la ración de la tarde.

En el caso del OvocontrolS $\AA$, el consumo del contraceptivo se registró en muy pocas aves $(20 \%)$ y cuando fue mezclado con maíz picado (OvocontrolS $\mathbb{\circledR}+$ maíz), su ingesta fue incluso menor. Se observó que, al transcurrir los días, las palomas comenzaron a seleccionar el maíz de la mezcla en detrimento del contraceptivo, lo que explica el aumento en las semanas 2 y 3 del consumo de la mezcla OvocontrolS $₫$ + maíz. Estos resultados coinciden parcialmente con lo registrado por Yoder et al., $\left(2006^{24}\right)$. En el mencionado estudio con patos, se midió el efecto en el consumo y la concentración de DNC en plasma al administrar la nicarbazina por cuatro métodos diferentes. Los autores encontraron que suministrar el producto con la comida, fue la forma menos efectiva.

En tanto, las aves utilizadas en los experimentos, fueron capturadas en un tambo y es posible que se encuentren familiarizadas con el alimento peleteado. Además, para el caso del OvoControlP® partido, las palomas fueron expuestas al contraceptivo evaluado, ofrecido junto con la ración de mantenimiento.

Los resultados de este estudio estarían indicando que, las palomas pueden tener un aprendizaje rápido a la hora de alimentarse con sus dietas preferidas. Este es un aspecto a tener en cuenta al diseñar e implementar la estrategia de administración de un contraceptivo oral en esta especie. Mezclarlo con un alimento de aspecto similar y/o apetecido por ellas, no necesariamente aumentaría su consumo. En cambio, generar un cebo con un tamaño de partícula preferido y ofrecerlo a poblaciones de palomas familiarizadas con este tipo de alimento peleteado, sí podría incrementar el consumo del contraceptivo. En este sentido, los establecimientos que alimenten ganado con pellets, podrían ser buenos candidatos para distribuir el cebo contraceptivo.

Con estos resultados, podemos concluir que el contraceptivo OvoControlP ${ }^{\circledR}$ partido fue aceptado y consumido por palomas torcazas en condiciones de cautiverio, en cantidades que potencialmente podrían causar un efecto en los parámetros reproductivos de la especie.

Futuros trabajos deberían evaluar si la dosis de nicarbazina utilizada en este estudio e ingerida por las palomas torcazas en cautiverio, efectivamente disminuye su éxito reproductivo.

\section{AGRADECIMIENTOS}

Los autores de este trabajo quieren agradecer al Instituto Nacional de Investigación Agropecuaria por brindar apoyo financiero para la realización de los trabajos mediante fondos INIA-FPTA. A la Mesa Tecnológica de Oleaginosos y a la Dirección General de Servicios Agrícolas por el apoyo logístico y edilicio. A la Agencia Nacional de Investigación e Innovación por financiar la beca de doctorado POS_NAC_2016_1_130028 de Lourdes Olivera. A los asistentes de laboratorio que colaboraron en la manutención de las aves. Al Dr. Michael Avery, Dr. Alexander MacDonald, Erick Wolf y a Scott Werner $(\mathrm{PhD})$ por sus aportes y sugerencias en la metodología. A la empresa Innolytics, LLC por donar el producto. 


\section{BIBLIOGRAFÍA}

1.- AVERY, M.L.; KEACHER, K.L. \& TILLMAN, E.A. 2006. Development of nicarbazin bait for managing rock pigeon populations. Vertebrate Pest Conference 22: 116-120.

2.- AVERY, M.L.; YODER, C.A. \& TILLMAN, E.A. 2008. Nicarbazin reduces reproduction by pigeons (Columba livia). Wildlife Res 35: 80-85

3.- AVERY, M.L.; YODER, C.A. \& TILLMAN, E.A.. 2008. Diazacon inhibits reproduction in invasive monk parakeet populations. Journal of wildlife management, 72 (6): $1449-1452$.

4.- BOMFORD, M. 1990. A Role for Fertility Control in Wildlife Management. Bulletin No. 7. Bureau of Rural Resources and the Australian Government Publishing Service, Canberra. 50 pp.

5.- BOU, N.; DARDANELLI, S.; OLIVERA, L.; TELLECHEA, G.; ORDUNA, L.A.; CANAVELLI, S. \& RODRÍGUEZ, E. 2016. Desarrollo de un método para evaluar el daño ocasionado por aves en cultivos comerciales de soja recién emergida. Idesia 34 (6): 67-74.

6.- BYNUM, K.; YODER, C.A.; EISEMANN, J.; JOHNSTON, J. \& MILLER, L.A. 2005. Development of nicarbazin as a reproductive inhibitor for resident Canada geese. Proc. of the Wildlife Damage Management Conf. 11: 179-189

7.- BYNUM, K.; EISEMANN, J.D.; WEAVER, G.C.; YODER, C.A.; FAGERSTONE, K.A. \& MILLER, L.A. 2007. Nicarbazin OvoControl G bait reduces hatchability of eggs laid by resident Canada geese in Oregon. J. Wildl. Manage 71 (1): 135-143.

8.- CALVO, M.V. 2006. Alimentación de la paloma torcaza (Zenaida auriculata) en relación con las prácticas agrícolas en Córdo- ba, Argentina. Tesis de Maestría en Manejo de Vida Silvestre. Universidad Nacional de Córdoba. 35p.

9.- DARDANELLI, S.; CALAMARI, N.C.; CANAVELLI, S.B. \& ZACCAGNINI, M.E. 2011. Biología de la paloma mediana (Zenaida auriculata), manchada (Patagioenas maculosa) y picazuro (Patagioenas picazuro). INTA EEA Paraná. Serie Extensión N ${ }^{\circ}$ 64:11-22.

10.- FAGERSTONE, K.A.; COFFEY, M.A.; CURTIS, P.D.; DOLBEER, R.A.; KILLIAN, G.J.; MILLER, L.A. \& WILMOT, L.M. 2002. Wildlife fertility control. Technical Review. The Wildlife Society, Bethesda, Maryland, USA. 30 pp.

11.- FAGERSTONE, K.A.; MILLER, L.A.; EISEMANN，J.D.; O'HARE，J.R. \& GIONFRIDDO, J.P. 2008. Registration for wildlife contraceptives in the United States of America, with Ovo-Control and GonaCon immunocontraceptive vaccines as examples. Wildlife Res. 35: 586 - 592.

12.- FAGERSTONE, K.A.; MILLER, L.A.; KILLIAN, G. \& YODER, C.A. 2010. Review of issues concerning the use of reproductive inhibitors, with particular emphasis on resolving human-wildlife conflicts in North America. Integ. Zool.1:15-30.

13.- FEARE, C.J. 1991. Bird pests in Argentina and Uruguay non-lethal control: chemosterilants. Unpublished report. Food and Agriculture Organization of the United Nations. 30 pp.

14.- KIRKPATRICK, J.F. \& TURNER, J.W. 1985. Chemical fertility control and wildlife management. Bioscience 35: 485-491.

15.- OLIVERA, L. \& RODRÍGUEZ, E. 2014. Aumentando el rendimiento de cultivos extensivos disminuyendo el daño de aves. Serie FPTA-INIA N ${ }^{\circ} 56$. Instituto Nacional de Investigación Agropecuaria. Montevideo, Uruguay. 44p. 
16.- OLIVERA, L.; TELLECHEA, G.; LAMANNA, A.; BANCHERO, G.E.; FERNÁNDEZ, E. \& RODRÍGUEZ , E.N. Sin publicar. Diagnóstico y cuantificación de la problemática causada por la concentración de aves en un tambo estabulado de Uruguay.

17.- ROGERS, E.F.; BROWN, R.D.; BROWN, J.E.; KAZAZIS, D.M.; LEANZA, W.J.; NICHOLS, J.R.; OSTLIND, D.A. \& RODINO, T.M. 1983.Nicarbazin complex yields dinitrocarbanilide as ultrafine crystals with improved anticoccidial activity. Science 222: 630-632.

18.- RODRÍGUEZ, E.; BRUGGERS, R.L.; BULLARD, R.W. \& COOK, R. 1995. An integrated strategy to decrease eared dove damage in sunflower crops. National Wildlife Research Center Repellents Conf. 1995. 409-421.

19.- RODRÍGUEZ, E. \& TISCORNIA, G. 2002. Evaluación de alternativas de control de la cotorra (Myiopsitta monachus). Serie FPTA - INIA No 8. Instituto Nacional de Investigación Agropecuaria. Montevideo, Uruguay. $48 \mathrm{p}$.

20.- RODRÍGUEZ, E.; TISCORNIA, G. \& OLIVERA, L. 2011. Disminución del daño por aves en pequeños predios. Serie FPTA-INIA N ${ }^{\circ} 29$. Instituto Nacional de Investigación Agropecuaria. Montevideo, Uruguay. $64 p$.
21.- RODRÍGUEZ, E. \& OLIVERA, L. 2017. Aumentando la protección de los cultivos de secano y feedlots al daño de aves mediante nuevas técnicas de repelencia. Reporte técnico proyecto INIA-FPTA 314.

22.- YODER, C.A.; ANDELT, W.F.; MILLER, L.A. \& GOODALL, M.J. 2004. Effectiveness of twenty, twenty-five diazacholesterol, avian gonadotropin-releasing hormone, and chicken riboflavin carrier protein for inhibiting reproduction in Coturnix quail. Poultry Science 83: 234 - 244.

23.- YODER, C.A.; MILLER, L.A. \& BYNUM, K.S. 2005. Comparison of nicarbazin absorption in chickens, mallards, and Canada geese. Poultry Science. J. 84: 1491-1494.

24.- YODER, C.A. \& MILLER, L.A. 2006. Avian contraceptive tools: one size does not fit all. Vertebrate Pest Conf.22: 110-115. 